PROCEEDINGS OF THE

AMERICAN MATHEMATICAL SOCIETY

Volume 127, Number 10, Pages 3095-3100

S 0002-9939(99)04876-5

Article electronically published on April 23, 1999

\title{
A MOORE SPACE WITH A $\sigma$-DISCRETE $\pi$-BASE WHICH CANNOT BE DENSELY EMBEDDED IN ANY MOORE SPACE WITH THE BAIRE PROPERTY
}

\author{
DAVID L. FEARNLEY
}

(Communicated by Alan Dow)

\begin{abstract}
The author answers a question raised in the literature about twenty five years ago and raised again more recently in Open Problems in Topology, by G. M. Reed, concerning the conjecture that every Moore space with a $\sigma$ discrete $\pi$-base can be densely embedded in a Moore space having the Baire property. Even though closely related results have made this conjecture seem likely to be true, the author shows that, surprisingly, the conjecture is false.
\end{abstract}

\section{INTRODUCTION}

A $\pi$-base for a space $S$ is a collection of open sets $P$ such that for any open set $U$ of $S$, there exists some $V \in P$ such that $V \subset U$. A $\sigma$-discrete $\pi$-base for $S$ is a $\pi$-base which is the countable union of discrete collections of open sets. A development for a space $S$ is a sequence of open coverings $G=\left(G_{1}, G_{2}, G_{3}, \ldots\right)$ of $S$ with the property that for any point $p$ and any open set $U$ of $S$ such that $p \in U$, there exists some positive integer $n$ such that if $g_{n} \in G_{n}$ and $p \in g_{n}$, then $g_{n}$ is contained in $U$. Without loss of generality we may also assume that $G_{n+1}$ is a subset of $G_{n}$ for all positive integers $n$. A Moore space is a regular Hausdorff space which has a development.

Moore spaces form a large and interesting class of topological spaces. For examples R. H. Bing [2, Example A] has constructed a Moore space such that the closure of every open set is non-metrisable, and M. E. Estill (Rudin) [3, Example 8] has constructed an example of a Moore space which is not completable. In such rather complicated cases it is useful to determine when there may exist relatively well behaved spaces which either are dense subspaces of the more complicated spaces, or are spaces into which the complicated spaces can be densely embedded. For instance, Reed [6] proved the surprising result that a Moore space $S$ has a $\sigma$-discrete $\pi$-base if and only if $S$ is densely embeddable in a developable Hausdorff space with the Baire property. Younglove [10] proved that every complete Moore space has a dense metrisable subspace, and Reed [5] proved that every completable Moore space also has a dense metrisable subspace. Fitzpatrick [4] proved that every Moore

Received by the editors June 23, 1997 and, in revised form, December 12, 1997.

1991 Mathematics Subject Classification. Primary 54D20, 54D25; Secondary 54 E52.

Key words and phrases. Moore space, $\sigma$-discrete $\pi$-base, Baire property, embedding.

This material is based on work supported under a National Science Foundation Graduate Fellowship. 
space with a $\sigma$-discrete $\pi$-base has a dense metrisable subspace. White [9] proved further that for a Moore space to have a dense metrisable subspace is equivalent to that Moore space having a $\sigma$-discrete $\pi$-base. Related results have also been obtained by K. E. Whipple [8] and J. M. Aarts and D. J. Lutzer [1].

The aforementioned results lead naturally to the question posed by Reed in [6] and [7] of whether or not it is true that a Moore space $S$ has a $\sigma$-discrete $\pi$-base if and only if $S$ can be embedded densely in a Moore space with the Baire property. In this paper the author answers this question by constructing a Moore space which has a $\sigma$-discrete $\pi$-base but cannot be densely embedded in a Moore space with the Baire property.

\section{Construction of the Counterexample}

Let $\mathbf{N}$ denote the set of positive integers, $\mathbf{Q}$ the set of rational numbers, and $\mathbf{R}$ the set of real numbers with the standard Euclidean metric, which metric we denote by $d$.

Let $L$ denote the irrational numbers. Let $\mathcal{Z}(L)$ denote the set of all subsets of $L$ of size $c$, the cardinality of the continuum. For each $n \in \mathbf{N}$ we let $\mathcal{C}_{n}$ be the set $\{n\} \times \mathcal{Z}(L) \times \mathbf{Q}$. Points of $S$ will be all elements of $L \cup(\mathbf{N} \times \mathcal{Z}(L) \times \mathbf{Q})$. If $W \in \mathcal{Z}(L)$ we will refer to $\{n\} \times\{W\} \times \mathbf{Q}$ as a "rational line" to which we will assign the standard Euclidean metric. We denote the rational line $\{n\} \times\{W\} \times \mathbf{Q}$ by $\mathbf{Q}_{(W, n)}$. We will refer to points of rational lines as "rational points." We will define the topology on $S$ by defining a development $G=\left(G_{1}, G_{2}, G_{3}, \ldots\right)$ for $S$. Three types of functions will be used in defining this development. First, for each $W \in \mathcal{Z}(L)$, let $g_{W}$ be a one-to-one function from $W$ onto $L$. Let $r_{(W, n)}$ be an order preserving and distance preserving function from $\mathbf{Q}_{(W, n)}$ onto $\mathbf{Q}$. Finally, let $f$ be a one-to-one map taking $L$ onto an almost disjoint collection $F$ of subsequences of the identity sequence on the set of positive integers (two members of $F$ have at most finitely many common terms) and for each $s \in L$ we denote by $f(s)(n)$ the $n$th member of $f(s)$.

We now define the development $G$. There are two types of open sets in $G_{n}$, $n \in \mathbf{N}$. First, let each open interval with diameter less than $1 / n$ on a rational line be an element of $G_{n}$. In order to describe the second type of open set we will define two additional terms. For each positive integer $m, W \in \mathcal{Z}(L)$ and $s \in W$, we define the set $I_{(W, m, s)}=r_{(W, m)}^{-1}\left(\mathbf{Q} \cap\left(g_{W}(s), g_{W}(s)+1\right)\right)$ and let $O_{m}(s)=$ $\bigcup\left\{I_{(W, m, s)} \mid s \in W \in \mathcal{Z}(L)\right\}$, the union as $W$ ranges over all elements of $\mathcal{Z}(L)$ containing $s$ as an element of the sets $I_{(W, m, s)}$. Then for each positive integer $j>n$ let $\{s\} \cup\left(\bigcup\left\{O_{f(s)(m)}(s) \mid m>j\right\}\right)$ be an element of $G_{n}$. Let $G_{n}$ be defined to contain exactly the elements defined above.

\section{Proof that $S$ is a Moore Space With a $\sigma$-Discrete $\pi$-BASE WHICH CANNOT BE DENSELY EMBEDDED IN ANY MOORE SPACE WITH THE BAIRE PROPERTY}

In order to facilitate the presentation of the results of this section we will divide them into two theorems.

Theorem 3.1. The space $S$ is a Moore space with a $\sigma$-discrete $\pi$-base.

Proof. The space $S$ is regular. Separation of rational points from closed sets by disjoint open sets is satisfied by the regularity of $\mathbf{Q}$. In the case of points of $L$, note 
that all the basic open development member sets containing a point of $L$ are both open and closed. Hence, regularity follows at these points also.

The space $S$ is Hausdorff. In the case of rational points this follows from the fact that $\mathbf{Q}$ is a Hausdorff space. Let $y \in L$, and let $x \in \mathcal{C}_{n}$. Elements of $G_{n}$ containing $x$ and $y$ are then disjoint. Let $x$ and $y$ be elements of $L$. Then $f(x)$ and $f(y)$ are elements of $F$. Thus $\{f(x)(i) \mid i \in \mathbf{N}\} \cap\{f(y)(i) \mid i \in \mathbf{N}\}$ is finite. We choose $m$ such that for positive integers $j, k>m, f(x)(j)$ is not equal to $f(y)(k)$. Then open sets in $G_{m}$ containing $x$ and $y$ are disjoint.

The space $S$ is developable. We must check that $G=\left(G_{1}, G_{2}, G_{3}, \ldots\right)$ is a development for $S$. Let $x$ be a point of $\mathcal{C}_{n}$, and let $U$ be an open set containing $x$. Choose a positive integer $m$ such that all the open sets on the rational line containing $x$ of diameter less than $1 / m$ are contained in $U$. Choose $k>m, n$. Then each element of $G_{k}$ containing $x$ is contained in $U$. Let $s$ be an element of $L$. Then let $V$ be an open set containing $s$, and let $j$ be a positive integer such that $O_{f(s)(n)}(s)$ is contained in $V$ for $n>j$. Then every open set in $G_{j}$ containing $s$ is contained in $V$.

The space $S$ has a $\sigma$-discrete $\pi$-base. Each $\mathcal{C}_{n}$ has a $\sigma$-discrete basis. Specifically, choose an ordered countable basis $B_{(W, n)}$ for $\mathbf{Q}_{(W, n)}$. Let $B_{(W, n, m)}$ denote the $m$ th element of $B_{(W, n)}$. Then for any pair of positive integers $n, m$ let $B(n, m)=$ $\left\{B_{(W, n, m)} \mid W \in \mathcal{Z}(L)\right\}$. Let $B=\bigcup\{B(n, m) \mid n, m \in \mathbf{N}\}$. Each $B(n, m)$ is discrete. This is because for $j>n$ no open set in $G_{j}$ containing any point of $L$ intersects any element of $B(n, m)$. Also, any rational point is contained in a rational line intersecting at most one element of $B(n, m)$. Hence, $B$ is $\sigma$-discrete. The elements for $B$ form a basis for $S-L$. Each open set containing a point of $L$ contains an open set in some $\mathcal{C}_{n}$ and hence an element of $B$. Hence, $B$ is a $\sigma$-discrete $\pi$-base for $S$.

Theorem 3.2. The space $S$ cannot be densely embedded in any Moore space with the Baire property.

Suppose by way of contradiction that $S$ is embedded densely in a Moore space $M$ with the Baire property. For each open set $V$ in $S$, we will denote by $M(V)$ the open set consisting of the union of all open sets of $M$ whose intersection with $S$ is contained in $V$.

Let $H=\left(H_{1}, H_{2}, H_{3}, \ldots\right)$, where $H_{n+1} \subset H_{n}$ for all positive integers $n$, be a development for $M$. For each rational line $\mathbf{Q}_{(W, n)}$, choose a cover $C_{W}(n, 1)$ of $\mathbf{Q}_{(W, n)}$ consisting of elements $V_{1}$ of $H_{1}$ such that the diameter of $V_{1} \cap S$ is less than one and $V_{1} \cap S$ is contained in $\mathbf{Q}_{(W, n)}$. Then choose a second cover $C_{W}(n, 2)$ of $\mathbf{Q}_{(W, n)}$ consisting of elements of $H_{2}$ such that, for each $V_{2} \in C_{W}(n, 2)$, the diameter of $V_{2} \cap S$ is less than $1 / 2$ and such that there exists $V_{1} \in C_{w}(n, 1)$ such that $V_{2} \subset V_{1}$ and $d\left(V_{2} \cap S, \mathbf{Q}_{(W, n)}-\left(V_{1} \cap S\right)\right)>0$. Choose a cover $C_{w}(n, 3)$ of $\mathbf{Q}_{(W, n)}$ such that $C_{W}(n, 3) \subset H_{3}$, and such that, for each $V_{3} \in C_{W}(n, 3)$, the diameter of $V_{3} \cap S$ is less than $1 / 3$ and there exists $V_{2} \in C_{W}(n, 2)$ such that $V_{3} \subset V_{2}$ and $d\left(V_{3} \cap S, \mathbf{Q}_{(W, n)}-\left(V_{2} \cap S\right)\right)>0$. Inductively, if $C_{W}(n, k)$ has been defined, then let $C_{W}(n, k+1)$ be a cover of $\mathbf{Q}_{(W, n)}$ which is a subset of $H_{k+1}$ such that, for each $V_{k+1} \in C_{W}(n, k+1)$, the diameter of $V_{k+1} \cap S$ is less than $1 /(k+1)$, and there exists $V_{k} \in C_{W}(n, k)$ such that $V_{k+1} \subset V_{k}$ and $d\left(V_{k+1} \cap S, \mathbf{Q}_{(W, n)}-\left(V_{k} \cap S\right)\right)>0$. Then for all $m, \bigcup C_{W}(n, m)$ is a dense open subset of $M\left(\mathbf{Q}_{(W, n)}\right)$. We know $\bigcup C_{W}(n, m)$ is dense in $M\left(\mathbf{Q}_{(W, n)}\right)$ because $S$ is dense in $M$, and hence in $M\left(\mathbf{Q}_{(W, n)}\right)$ and we know $M\left(\mathbf{Q}_{(W, n)}\right) \cap S=\mathbf{Q}_{(W, n)}$, which is contained in $\bigcup C_{W}(n, m)$. We know 
$\bigcup C_{W}(n, m)$ is contained in $M\left(\mathbf{Q}_{(W, n)}\right)$ because for each $V_{m} \in C_{W}(n, m), V_{m} \cap S \subset$ $\mathbf{Q}_{(W, n)}$. Hence the intersection over all $m$ of such sets, $\bigcap\left\{\bigcup C_{W}(n, m) \mid m \in \mathbf{N}\right\}$, is a dense $G_{\delta}$ subset of $M\left(\mathbf{Q}_{(W, n)}\right)$. For each rational line $\mathbf{Q}_{(W, n)}$, we will denote $C_{(W, n)}=\bigcap\left\{\bigcup C_{W}(n, m) \mid m \in \mathbf{N}\right\}$.

We will define a one-to-one function $e_{(W, n)}$ from $C_{(W, n)}$ into $\mathbf{R}$ by defining $e_{(W, n)}(q)=r_{(W, n)}(q)$ for all $q \in \mathbf{Q}_{(W, n)}$, and defining $e_{(W, n)}(p)$ for $p \in C_{(W, n)}-$ $\mathbf{Q}_{(W, n)}$ to be the point of convergence of a Cauchy sequence in $\mathbf{R}$ corresponding to a Cauchy sequence in $\mathbf{Q}_{(W, n)}$ converging to $p$. We will now define a particular Cauchy sequence in $\mathbf{R}$ which will converge to $e_{(W, n)}(p)$. Let $U$ be an open set containing $p$ whose intersection with $S$ does not contain all of $\mathbf{Q}_{(W, n)}$. We claim that for some positive integer $m$ there exists $h_{m} \in H_{m}$ with $p \in h_{m}$, so that $h_{m} \cap S$ is contained in $\mathbf{Q}_{(W, n)}$, has diameter less than $1 / m$, and has the property that $d\left(h_{m} \cap S, \mathbf{Q}_{(W, n)}-U\right)>0$. We now prove this claim. Choose $j$ such that all $h_{j} \in H_{j}$ containing $p$ are contained in $U$. We choose $V_{j+1} \in C_{W}(n, j+1)$ such that $p \in V_{j+1}$. Then we know $V_{j+1}$ is contained in $h_{j}$ for some $h_{j} \in C_{W}(n, j)$ such that $d\left(V_{j+1} \cap S, \mathbf{Q}_{(W, n)}-\left(h_{j} \cap S\right)\right)>0$ by construction. Hence, $d\left(V_{j+1} \cap S, \mathbf{Q}_{(W, n)}-U\right)>$ 0 , and letting $m=j+1$ in the statement of the claim, $V_{j+1}$ is an element of $H_{j+1}$ satisfying all the criteria we required and the proof of the claim is complete.

Then for each point $p$ of $C_{(W, n)}$ choose a collection $h(1, p), h(2, p), \ldots$ of increasingly indexed development member open sets containing $p$ such that each $h(1, p) \cap S$ is contained in $\mathbf{Q}_{(W, n)}$ and has diameter less than one, and such that for all positive integers $k, d\left(h(k+1, p) \cap S, \mathbf{Q}_{(W, n)}-(h(k, p) \cap S)\right)>0$, and furthermore the diameters of the $h(k, p) \cap S$ converge to zero. We can do this by the claim, letting $U$ be $h_{k}$ repeatedly. Note that each $h(k, p) \cap S$ is bounded. Since the set of all points of $\mathbf{Q}_{(W, n)}$ greater than (or respectively less than) all points of $h(m, p) \cap S$ is contained in $\mathbf{Q}_{(W, n)}-(h(m, p) \cap S)$, this set has positive distance from $h(m+1, p) \cap S$.

Thus, we know that there exist points of $h(m, p) \cap S$ which are greater than (or respectively less than) and are a positive distance from all elements of $h(m+1, p) \cap S$ for each positive integer $m$. Choose two rational points from each $h(k, p), p_{\mathbf{Q}}(2 k-1)$ and $p_{\mathbf{Q}}(2 k)$, so that $p_{\mathbf{Q}}(2 k-1)$ is less than and a positive distance from all points of $h(k+1, p)$ and $p_{\mathbf{Q}}(2 k)$ is greater than and a positive distance from all elements of $h(k+1, p) \cap S$. Because the diameters of the $h(k, p) \cap S$ converge to zero, the sequence $\left(p_{\mathbf{Q}(m)}\right)$ corresponding to $p$ is a Cauchy sequence. Then $\left(e_{(W, n)}\left(p_{\mathbf{Q}}(m)\right)\right)=\left(r_{(W, n)}\left(p_{\mathbf{Q}}(m)\right)\right)$ is also a Cauchy sequence, and converges in $\mathbf{R}$ to the point we define to be $e_{(W, n)}(p)$. We also define a map $e_{\mathbf{Q}(W, n)}$ taking each point $p$ in $C_{(W, n)}-\mathbf{Q}_{(W, n)}$ to its corresponding chosen Cauchy sequence $\left(p_{\mathbf{Q}(m)}\right)$ as defined above.

We wish to show $e_{(W, n)}$ is one to one. Let $x, y \in C_{(W, n)}-\mathbf{Q}_{(W, n)}$. Then there are disjoint open sets $V(x)$ and $V(y)$ containing $x$ and $y$ respectively. By our claim there are open sets $U(x)$ and $U(y)$ containing $x$ and $y$ respectively such that $d\left(U(x) \cap S, \mathbf{Q}_{(W, n)}-V(x)\right)>0$ and $d\left(U(y) \cap S, \mathbf{Q}_{(W, n)}-V(y)\right)>0$. We let $\left(x_{\mathbf{Q}}(m)\right)=e_{\mathbf{Q}(W, n)}(x)$ and $\left(y_{\mathbf{Q}}(m)\right)=e_{\mathbf{Q}(W, n)}(y)$. We choose a positive integer $j$ so that for all $m>j, x_{\mathbf{Q}}(m) \in U(x) \cap S$ and $y_{\mathbf{Q}}(m) \in U(y) \cap S$. Then the set of all members of the sequence $\left(e_{(W, n)}\left(x_{\mathbf{Q}}(m)\right)\right)$, such that $m>j$, is a positive distance from the set of all members of the sequence $\left(e_{(W, n)}\left(y_{\mathbf{Q}}(m)\right)\right)$, such that $m>j$, and hence these sequences converge to different points $e_{(W, n)}(x)$ and $e_{(W, n)}(y)$ in $\mathbf{R}$. Suppose $x \in \mathbf{Q}_{(W, n)}$ and $y \in C_{(W, n)}-\mathbf{Q}_{(W, n)}$. Then similarly we choose a positive integer $k$ such that the set of all members of $\left(y_{\mathbf{Q}}(m)\right)$, such that $m>k$, 
has a positive distance from $x$. Then we know that the set of all members of $\left(e_{(W, n)}\left(y_{\mathbf{Q}}(m)\right)\right)$, such that $m>k$, has a positive distance from $e_{(W, n)}(x)$ and hence $\left(e_{(W, n)}\left(y_{\mathbf{Q}}(m)\right)\right)$ converges to a point $e_{(W, n)}(y)$ in $\mathbf{R}$ which is not equal to $e_{(W, n)}(x)$. Hence, $e_{(W, n)}$ is one to one.

Since $M$ is regular we note that for each point $s$ of $L$ we may choose open sets $U_{1}(s)$ and $U_{2}(s)$ in $M$ containing $s$ such that $U_{1}(s)$ contains the closure of $U_{2}(s)$ and such that for $i=1,2, U_{i}(s) \cap S$ is an element of $G_{i}$ containing $s$. Assume we an choose positive integers $i$ and $m$ such that $U_{i}(s)$ contains points of $\mathcal{C}_{m}$. Assume further that we can choose $W \in \mathcal{Z}(L)$ such that $e_{(W, m)}\left(C_{(W, m)}\right)$ contains $g_{W}(s)$. Then we will denote by $t$ the point of $C_{(W, m)}$ such that $e_{(W, m)}(t)=g_{W}(s)$. We will show that then $t$ is a limit point of $U_{i}(s)$ which is not contained in $U_{i}(s)$.

Let $O$ be an open set containing $t$. Let $\left(t_{\mathbf{Q}}(n)\right)=e_{\mathbf{Q}(W, m)}(t)$. Then we may pick $j$ so that for all $i, k>j, d\left(t_{\mathbf{Q}}(i), t_{\mathbf{Q}}(k)\right)<1$ and $t_{\mathbf{Q}}(k)$ is contained in $O \cap \mathbf{Q}_{(W, m)}$. Since $e_{(W, m)}$ is distance and order preserving on all members of $\left(t_{\mathbf{Q}}(n)\right)$, we know that $e_{(W, m)}(t)<e_{(W, m)}\left(t_{\mathbf{Q}}(2 j)\right)$ and $e_{(W, m)}\left(t_{\mathbf{Q}}(2 j+1)\right)<e_{(W, m)}(t)$. Note also that $d\left(e_{(W, m)}\left(t_{\mathbf{Q}}(2 j)\right), e_{(W, m)}(t)\right)<d\left(e_{(W, m)}\left(t_{\mathbf{Q}}(2 j)\right), e_{(W, m)}\left(t_{\mathbf{Q}}(2 j+1)\right)\right)<1$. Hence, we know that

$$
t_{\mathbf{Q}}(2 j) \in r_{(W, m)}^{-1}\left(\mathbf{Q} \cap\left(g_{W}(s), g_{W}(s)+1\right)\right) .
$$

Thus we have shown that every open set $O$ containing $t$ contains a point of $r_{(W, m)}^{-1}\left(\mathbf{Q} \cap\left(g_{W}(s), g_{W}(s)+1\right)\right)$. Recall that $U_{i}(s)$ contains an element of $G_{i}$, and by the definition of $G_{i}$ every element of $G_{i}$ containing points of $\mathcal{C}_{m}$ and the point $s$ contains $r_{(W, m)}^{-1}\left(\mathbf{Q} \cap\left(g_{W}(s), g_{W}(s)+1\right)\right)$. Hence, $t$ is a limit point of $U_{i}(s)$. Note also that $t_{\mathbf{Q}}(2 j+1)$ is not an element of $r_{(W, m)}^{-1}\left(\mathbf{Q} \cap\left(g_{W}(s), g_{W}(s)+1\right)\right)$, and thus we have shown that every open set containing $t$ contains points of $\mathcal{C}_{m}$ which are not contained in any element of $G_{i}$ which contains $s$, and hence contains points which are not contained in $U_{i}(s)$. Thus we know that $t$ is not contained in $U_{i}(s)$. Hence, we have the desired result that for any positive integers $i$ and $m$, if $U_{i}(s)$ contains points of $\mathcal{C}_{m}$, and we can choose $W \in \mathcal{Z}(L)$ such that $e_{(W, m)}\left(C_{(W, m)}\right)$ contains $g_{W}(s)$, then the point $t$, such that $e_{(W, m)}(t)=g_{W}(s)$, is a limit point of $U_{i}(s)$ and is not contained in $U_{i}(s)$.

The closure of $U_{2}(s)$ is contained in $U_{1}(s)$. We claim that for all $m$, if $U_{2}(s)$ contains points of $\mathcal{C}_{m}$, then there does not exist $W \in \mathcal{Z}(L)$ such that $e_{(W, m)}\left(C_{(W, m)}\right)$ contains $g_{W}(s)$. Suppose by way of contradiction that $U_{2}(s)$ contains points of $\mathcal{C}_{m}$ and we may choose $W \in \mathcal{Z}(L)$ such that $e_{(W, m)}\left(C_{(W, m)}\right)$ contains $g_{W}(s)$. If $U_{2}(s)$ contains points of $\mathcal{C}_{m}$, then $U_{1}(s)$ contains points of $\mathcal{C}_{m}$. As before we let $t$ be the point of $C_{(W, m)}$ such that $e_{(W, m)}(t)=g_{W}(s)$. Then we have shown that $t$ is not contained in $U_{1}(s)$ and is a limit point of $U_{2}(s)$, and this contradicts the fact that the closure of $U_{2}(s)$ is contained in $U_{1}(s)$. Hence, the result follows that for all $m$, if $U_{2}(s)$ contains points of $\mathcal{C}_{m}$, then there does not exist $W \in \mathcal{Z}(L)$ such that $e_{(W, m)}\left(C_{(W, m)}\right)$ contains $g_{W}(s)$.

Since $U_{2}(s)$ contains some element of $G_{2}$, we may choose a positive integer $i>2$ such that for all positive integers $n>i, U_{2}(s)$ contains points of $\mathcal{C}_{f(s)(n)}$. Hence, for each point $s$ of $L$, we may choose a positive integer which we will denote by $m(s)$ such that for any integer $n>m(s), U_{2}(s)$ contains points of $\mathcal{C}_{f(s)(n)}$, and hence there does not exist any set $W \in \mathcal{Z}(L)$ such that $e_{(W, f(s)(n))}\left(C_{(W, f(s)(n))}\right)$ contains $g_{W}(s)$. Then choose a set $P$ contained in $L$ and a positive integer $j$, such that $P$ has cardinality $c$ and $j=f(x)(m(x))$ for each $x \in P$. This can be done because 
there are only countably many positive integers and $c$ many elements $s$ of $L$, and each such $s$ has associated with $s$ some positive integer $m(s)$. Then choose a set $J$ contained in $P$ and a positive integer $k>j$ such that $J$ has cardinality $c$ and for each $z \in J$ there exists some positive integer $n$ such that $k=f(z)(n)$. We know such a $J$ exists because there are $c$ many elements $x$ of $P$ and for each such $x$ there exists an associated $f(x)(n)$ such that $f(x)(n)>j$.

Since $J$ has cardinality $c$ and is a subset of $L$ there is a rational line $\mathbf{Q}_{(J, k)}$. We wish to show that $C_{(J, k)}-\mathbf{Q}_{(J, k)}$ is empty. Suppose $C_{(J, k)}-\mathbf{Q}_{(J, k)}$ is not empty. Then let $p$ be an element of $C_{(J, k)}-\mathbf{Q}_{(J, k)}$. Since $e_{(J, k)}$ is one to one and maps $\mathbf{Q}_{(J, k)}$ onto the rational numbers $\mathbf{Q}$ in $\mathbf{R}$, we know that $e_{(J, k)}(p) \in L$. Since $g_{J}$ maps $J$ onto $L$, we may choose $z \in J$ such that $g_{J}(z)=e_{(J, k)}(p)$. But since $z$ is also an element of $P$ and $k>j$, we know that $k>f(z)(m(z))$, and hence since $k$ is also a member of $f(z), k=f(z)(n)$ for some positive integer $n$ greater than $m(z)$, so there does not exist any set $W$ contained in $L$ such that $W$ has cardinality $c$ and such that $e_{(W, k)}\left(C_{(W, k)}\right)$ contains $g_{W}(z)$. But this is a contradiction to the fact that $J$ is a subset of $L$ of cardinality $c, g_{J}(z)=e_{(J, k)}(p)$, and $p \in C_{(J, k)}$, because then $e_{(J, k)}\left(C_{(J, k)}\right)$ contains $e_{(J, k)}(p)=g_{J}(z)$. Hence, we know that $C_{(J, k)}-\mathbf{Q}_{(J, k)}$ is empty.

We know that $C_{(J, k)}$ is a dense $G_{\delta}$ subset of $M\left(\mathbf{Q}_{(J, k)}\right)$, and from the preceding argument we know that $C_{(J, k)}=\mathbf{Q}_{(J, k)}$. But no homeomorphic image of $\mathbf{Q}$ is a dense $G_{\delta}$ subset of any Baire space.

Hence, $S$ cannot be densely embedded in a Moore space with the Baire property.

\section{REFERENCES}

1. J. M. Aarts and D. J. Lutzer, Pseudo-completeness and the product of Baire spaces, Pacific J. Math. 48 (1), (1973), 1-10. MR 48:5009

2. R. H. Bing, Metrization of topological spaces, Canad. J. Math. 3 (1951), 175-186. MR 13:264f

3. M. E. Estill (Rudin), Concerning abstract spaces, Duke Math. J. 17 (1950), 317-327. MR 13:148b

4. B. Fitzpatrick, On dense subsets of Moore spaces II, Fund. Math. 61 (1967), 91-92. MR 36:2119

5. G. M. Reed, Concerning completable Moore spaces, Proc. Amer. Math. Soc. 36 (1972), 591596. MR 46:8185

6. G. M. Reed, Lecture notes in mathematics, vol. 378, Springer-Verlag, 1972, pp. 368-384.

7. J. Van Mill and G. M. Reed (editors), Open problems in topology, North-Holland Publ., 1990, problem 303. MR 92c:54001

8. K. E. Whipple, Cauchy sequences in Moore spaces, Pacific J. Math. 18 (1966), 191-199. MR 33:4889

9. H. E. White, Jr., First countable spaces that have pseudo-bases, Canad. J. Math. Bull. 21 (1978), 103-112. MR 58:2675

10. J. N. Younglove, Concerning dense metric subspaces of certain non-metric spaces, Fund. Math. 48 (1959), 15-25. MR 22:1878

Mathematics Institute, 24-29 St. Giles, Oxford University, Oxford OX1 3LB, EngLAND

E-mail address: david.fearnley@st-edmund-hall.oxford.ac.uk

Current address: Department of Mathematics, Brigham Young University, Provo, Utah 84602

E-mail address: davidf@math. byu.edu 